\title{
Aus der § 64 LFGB-Arbeitsgruppe MALDI-TOF: Leitlinien für die Validierung von Spezies-Identifizierungen mittels MALDI-TOF-MS
}

\section{From the $\S 64$ LFGB MALDI-TOF working group: guidelines for validation of species identifications with MALDI-TOF-MS}

\author{
Jörg Rau ${ }^{1}\left[\right.$ ] Tobias Eisenberg $^{2} \cdot$ Christine Wind $^{3} \cdot$ Ingrid Huber ${ }^{4} \cdot$ Melanie Pavlovic $^{4} \cdot$ René Becker $^{5}$
}

Received: 26 July 2021 / Revised: 21 September 2021 / Accepted: 23 September 2021 / Published online: 1 December 2021 (C) The Author(s) 2021

\section{Zusammenfassung}

Bei der Untersuchung von Lebensmitteln ist die matrixunterstützte Laser-Desorptions-/Ionisations-Flugzeit-Massenspektrometrie (MALDI-TOF-MS) zur Identifizierung von Mikroorganismen vielfach im Einsatz. Weitere Anwendungen wie die Tierartbestimmung aus Fleisch, Milch oder Fisch kommen hinzu. Vorgaben für die Validierung von Spezies-Identifizierungen fehlen bisher. Die nach § 64 LFGB am Bundesamt für Verbraucherschutz und Lebensmittelsicherheit eingerichtete Arbeitsgruppe „MALDI-TOF“ hat nun in einer Leitlinie allgemein gefasste Empfehlungen zur Validierung zusammengestellt. Diese richten sich an Einzellabore oder Laborverbünde und zeigen praxisnah Möglichkeiten, qualitative MALDI-TOF-MSMethoden zu überprüfen. Die besonderen Chancen der Technologie, wie die Verwendung individuell zusammengestellter Referenz-Datenbanken und die Nutzung von Sammlungen gut dokumentierter Einzelspektren zur Validierung, werden in der hier vorgestellten Leitlinie berücksichtigt.

Schlüsselwörter MALDI-TOF-MS · Validierung · Identifizierung · Leitlinie

\begin{abstract}
Matrix-assisted laser-desorption/ionization-time-of-flight-mass-spectrometry (MALDI-TOF-MS) is widely used to identify microorganisms. Recently, new applications such as identification of the animal species from meat, milk or fish are emerging. Standards for the validation of species identifications are still missing. Now, the § 64-LFGB working-group "MALDI-TOF", established at the Federal Office of Consumer Protection and Food Safety, has compiled a guideline for the validation of species identifications. This guideline is intended for single laboratories as well as for lab networks and shows practical ways for validation of qualitative MALDI-TOF-MS methods. The special opportunities of the technology, in particular the use of extended reference databases and of collections of well-documented individual spectra for validation, have been taken into account in the guideline presented.
\end{abstract}

Keywords MALDI-TOF-MS · Validation · Identification · Guidelines

Jörg Rau

joerg.rau@cvuas.bwl.de

1 Chemisches und Veterinäruntersuchungsamt Stuttgart (CVUAS), Fellbach, Deutschland

2 Landesbetrieb Hessisches Landeslabor (LHL), Gießen, Deutschland
3 Chemisches und Veterinäruntersuchungsamt Freiburg, Freiburg, Deutschland

4 Bayerisches Landesamt für Gesundheit und Lebensmittelsicherheit, Oberschleißheim, Deutschland

5 Bundesamt für Verbraucherschutz und Lebensmittelsicherheit, Berlin, Deutschland 


\section{Einleitung}

Moderne Analyseverfahren haben ein großes Potential, die Sicherheit und Authentizität von Lebensmitteln zu überwachen. Standardisierung und transparente Validierung sind wichtige Voraussetzungen für den Einsatz in der akkreditierten Umgebung der amtlichen Lebensmittelüberwachung. Die Aufnahme neuer analytischer Verfahren in die „Amtliche Sammlung von Verfahren zur Probenahme und zur Untersuchung" (ASU) ist ein geeignetes Mittel, um ein hohes Maß an Anerkennung der Methoden sicherzustellen.

Nach einem ersten Kick-off Workshop zu neuen Analysentechniken im Jahr 2016 folgte auf Initiative des Bundesamtes für Verbraucherschutz und Lebensmittelsicherheit (BVL) die Gründung mehrerer Arbeitsgruppen nach $\S 64$ des Lebensmittel- und Futtermittelgesetzbuches (LFGB) mit neuen thematischen Schwerpunkten (Szabo et al. 2017). Im Zuge dessen konstituierte sich im Februar 2019 die Arbeitsgruppe „MALDI-TOF“.

Die MALDI-TOF-MS wird in amtlichen Laboren der Lebensmittel- und Futtermittelüberwachung Deutschlands für die Identifizierung von Mikroorganismen bereits weithin genutzt (Malorny et al. 2020). Weitere Anwendungen zur Untersuchung der Authentizität, wie die Spezies-Identifizierung bei Fleisch, Fisch, Milchprodukten oder Pilzen, werden zunehmend erschlossen (Pavlovic et al. 2020; Rau et al. 2020, 2021; Stahl und Schröder 2017). Allerdings fehlte es bisher an spezifischen Normvorgaben für diese Analysentechnik, so dass auch im Umfeld der Akkreditierung unterschiedliche Vorgehensweisen zur Validierung oder Verifizierung diskutiert wurden. Bei den Laboren bestand daher ein großer Bedarf für ein standardisiertes Vorgehen bei der Validierung von MALDI-TOF-MS-Verfahren (Szabo et al. 2017).

Die neue Arbeitsgruppe „MALDI-TOF“ hat daher eine erste allgemein gefasste Leitlinie zur Validierung von MALDI-TOF-MS-Methoden erstellt und kürzlich veröffentlicht (BVL 2021). Die Leitlinie beschreibt minimale Leistungskriterien, die bei einer Validierung für identifizierende MALDI-Methoden im Einzellabor (unabhängig arbeitendes Labor mit einem Standort) oder in einem zusammenarbeitenden Laborverbund (mehrere Standorte, definierte Arbeitsgemeinschaft, gemeinsamer Pool an Referenzspektren und Validierungsspektren) zu berücksichtigen sind.

\section{Technik}

Mit dem Identifizierungsverfahren MALDI-TOF-MS werden Masse-Ladungsmuster (Massenspektren) von Biomolekülen (beispielsweise Proteinen) einer Probe erzeugt. Die erhaltenen Massenspektren werden mit in Datenbanken hinterlegten bekannten Referenzmustern verglichen. Durch ein computergestütztes Ranking der besten Übereinstimmungen mit den Referenzmustern und der Anwendung von empirischen Entscheidungsregeln erfolgt die Zuordnung der Probe und deren Identifizierung. Mit den meisten Verfahren ist eine Identifizierung der Probe nur möglich, wenn in den Datenbanken auch entsprechend passende Referenzmuster enthalten sind. Die Datenbanken sind somit eine Kernkomponente des Verfahrens. Methoden zur Identifizierung können beispielsweise für die Genus-, die Spezies-, die Subspeziesebene oder für einzelne Eigenschaften, wie den Nachweis einer Toxin-Bildung, erarbeitet werden (Pavlovic et al. 2013; Stahl und Schröder 2017; Rau et al. 2020; Rizzardi et al. 2013; Ulrich et al. 2019).

Manche Systeme am Markt sind mit einer geschlossenen Datenbank ausgestattet, die a priori seitens der Herstellerfirma validiert ist. Eine Veränderung der Datenbanken durch den Nutzer/die Nutzerin ist in diesen Fällen nicht vorgesehen. Der besondere Vorteil der MALDI-TOF-MS resultiert allerdings auch aus der Möglichkeit, durch neue Referenzspektren schnell auf aktuelle Anforderungen oder diagnostische Lücken in der Untersuchung von Lebensmitteln und Futtermitteln oder in der medizinischen Mikrobiologie zu reagieren. Daraus folgt als besondere Herausforderung, diese nun dynamischen Datenbanken auch schlüssig zu überprüfen, beziehungsweise Regeln für wiederholte Überprüfungen zu definieren. Dies gilt vor allem, wenn im Labor ,in-house“ "Datenbanken verwendet werden oder diese Datenbanken für neue Anwendungen erweitert werden sollen. Eine weitere große Herausforderung besteht darin, dass die Identifizierung mangels adäquater Alternativen bisher fast ausschließlich mithilfe von kommerziellen Algorithmen erfolgt, bei denen meist nicht genau bekannt ist, wie die Kennzahlen zur Identifizierung (Score-Werte, Prozentränge) ermittelt wurden.

Ein Weg der Validierung solcher „Black-Box“-Systeme aus Gerät, Datenbank und Software ist eine parameterbezogene Vorgehensweise auf Basis der Resultate für definierte Isolate oder Materialien. Neben selbst erzeugten Spektren können dazu systematisch auch Massenspektren anderer Nutzer/Nutzerinnen in die Validierung einbezogen werden. Als Voraussetzung dafür müssen diese externen Massenspektren dieselben stringenten Anforderungen hinsichtlich technischer Qualität und Transparenz der Dokumentation wie die kommerziellen Einträge oder die im eigenen Labor aufgenommenen Spektren erfüllen. Mit einem solchen nutzerübergreifenden Ansatz, bei welchem nicht die Isolate selbst, sondern lediglich deren elektronische Spektren ausgetauscht werden müssen, kann die zur robusten Validierung erforderliche Anzahl an Stichproben im Labor auch für seltenere Materialien leichter erreicht werden. Zudem ist die Übertragbarkeit der Ergebnisse einfach und eröffnet damit eindrucksvolle und nahezu unbegrenzte Chancen für global 
kooperierende Laborverbünde. Ein transparenter Austausch von Einzelspektren zu Validierungszwecken kann einfach per E-Mail initiiert werden und wird beispielsweise über die offene MALDI-User Plattform MALDI-UP gefördert (https://maldi-up.ua-bw.de; Rau et al. 2016).

\section{Validierungszweck}

Validierungen sollen die Gebrauchstauglichkeit der betreffenden Methoden für den vorgesehenen Zweck belegen. MALDI-TOF-MS wird insbesondere als identifizierendes qualitatives Verfahren verwendet. Anders als bei qualitativen Verfahren, die mit definierten Nachweisgrenzen die Ab- oder Anwesenheit des zu bestimmenden Parameters anzeigen, liegt bei einem identifizierenden Verfahren das Probenmaterial in ausreichender Menge vor. Als methodische Aufgabe steht die Benennung des vorliegenden Materials oder des mikrobiellen Isolates auf einer taxonomischen Ebene (beispielsweise Subspezies, Spezies, Genus, Familie) oder die Bestimmung der Zugehörigkeit zu einer definierten Gruppe (beispielsweise Toxinbildner) im Vordergrund. Validiert wird der Ziel-Parameter für die im konkreten Laborkontext übliche Untersuchungsaufgabe (beispielsweise die Identifizierung der Spezies Staphylococcus aureus bei Isolaten aus Lebensmitteln und Proben der veterinärmedizinischen Bakteriologie; oder die Tierart Büffel bei Mozzarella). Aus der jeweiligen Aufgabe leitet sich die Vorgehensweise bei der Validierung einer MALDITOF-MS-Methode ab.

In den Leitlinien werden Leistungskriterien für Validierungen der MALDI-TOF-MS aus drei unterschiedlichen Anwendungen beschrieben: (1) die nicht gerichtete Identifizierung (Screening), (2) die Identifizierung konkreter Parameter sowie (3) die Bestätigungsprüfung für konkrete Parameter. Angepasst an die jeweilige Anwendung werden, neben spezifischen Stichprobengrößen, Kriterien für die Richtig-positiv-Rate (Inklusivität), die Falsch-negativ-Rate, die Richtig-negativ-Rate (Exklusivität), die Falsch-positivRate, oder-bei Verwendung im Screening-die Korrektklassifikationsrate sowie die Robustheit vorgeschlagen. In den Anhängen der Leitlinie werden Beispiele für alle Anwendungen gegeben (BVL 2021).

\section{Anwendungen}

\subsection{Nicht gerichtete Identifizierung (Screening)}

Die Validierung einer nicht gerichteten Identifizierung (Screening) überprüft, ob der beabsichtigte Ziel-Parameter für ein Arbeitsgebiet in der üblichen (meist taxonomischen) Bandbreite generell richtig erfasst wird.

Den Empfehlungen nach werden beim Screening für die Berechnung nur gelungene Identifizierungsentscheidungen des MALDI-TOF-MS-Systems als Stichprobe berücksichtigt. Somit zeigt sich in der experimentellen Überprüfung der Anteil der mit dem Verfahren richtig identifizierten Materialien oder Isolate bezogen auf alle identifizierten Proben. Als umfassende Kennzahl wird die Korrektklassifikationsrate errechnet (Trevetan 2017). Üblicherweise ist die Anzahl der zur Validierung eingesetzte Stichproben für das Screening hoch $(>500)$. Die beabsichtigten Parameter(-gruppen) müssen in der experimentellen Überprüfung in sinnvoller Gewichtung vertreten sein. Somit wird die Abdeckung des MALDITOF-MS-Systems für das vorgesehene Arbeitsgebiet demonstriert.

\subsection{Zielgerichtete Identifizierung konkreter Parameter}

Die Validierung einer (ziel-)gerichteten identifizierenden MALDI-TOF-MS-Methode fokussiert eine einzelne konkrete Identifizierungsentscheidung, den Ziel-Parameter. Dies kann beispielsweise eine Mikroorganismenspezies, eine Tierart oder einer Pilzgattung sein.

Bei der zielgerichteten Identifizierung konkreter Parameter werden, wie beim Screening, für die Berechnung ebenfalls nur gelungene Identifizierungsentscheidungen als Stichprobe berücksichtigt.

\subsection{Bestätigungsprüfung}

Auch die Validierung einer bestätigenden MALDI-TOFMS-Methode zielt auf eine einzelne Identifizierungsentscheidung (einen Ziel-Parameter) ab. Bei einer Bestätigungsprüfung soll für den Parameter aber auch im negativen Fall eine eindeutige Aussage möglich sein.

Für die Bestätigungsprüfung wird daher empfohlen, für die Berechnung die Identifizierungsentscheidungen aller Proben als Stichprobe zu berücksichtigen. Nicht identifizierte Proben des Ziel-Parameters werden grundsätzlich als falsch-negativ gewertet. In der experimentellen Überprüfung zeigt sich der Anteil der mit dem Verfahren richtig identifizierten Materialien/Isolate des Ziel-Parameters, bezogen auf alle Proben.

Für die experimentelle Überprüfung der zielgerichteten Identifizierung konkreter Parameter und der Bestätigungsprüfung werden minimale Stichprobengrößen unabhängiger Spektren für den Ziel-Parameter und für die 
Nicht-Ziel-Parameter, angelehnt an die Normenreihe DIN EN ISO 16140 (2016) angegeben. Bei Nichterreichen der jeweiligen Vorgaben zur Richtig-positiv-Rate, der Richtignegativ-Rate, der Falsch-negativ- oder der Falsch-positivRate können Einschränkungen in der Interpretierbarkeit der Identifizierung die Folge sein. In diesen Fällen ist eine Absicherung durch eine unabhängige geeignete (beispielsweise molekularbiologische) Methode anzuraten.

\section{Ausblick}

Die in den Leitlinien dargestellte Vorgehensweisen für die drei verschiedenen Anwendungen der MALDI-TOF-MS stellen Empfehlungen dar, die sich unter anderem in der Praxis der Untersuchung von Lebensmitteln und Futtermitteln bewährt haben. Die Darstellung der an verschiedene Anwendungszwecke angepassten flexiblen Validierungskonzepte soll es Personen, die MALDI-TOF-MS nutzen, ermöglichen, ergänzte Datenbanken und neue Verfahren, beispielsweise zur Artbestimmung von Tieren standardisiert und gleichartig zu überprüfen. Durch laborinterne oder zwischen Laboratorien durchgeführte Studien zur Validierung unter Anwendung dieser Leitlinie steht die entsprechende Methode im amtlichen Labor im Einklang mit Art. 34 der VO (EU) 2017/625 (2017)

Die durch Anwendung der Leitlinie ermittelten Validierungsdaten sollen zudem bei der Entscheidung helfen, ob und wie die betreffenden Methoden in einem Ringversuch validiert werden können. Dies ist Voraussetzung für eine mögliche Aufnahme in die Amtliche Methodensammlung des BVL. Für Ringversuche wurde daher ein allgemeines Schema in die Leitlinie integriert, das bisherige Erfahrungen im Laborvergleich berücksichtigt (Huber et al. 2018). Die Planung und Durchführung von laborübergreifenden Validierungsstudien im mikrobiologischen Bereich gehört zu den nächsten Schritten der $\S 64$ LFGB-Arbeitsgruppe.

Zur Etablierung eines einheitlichen Vorgehens bei der Validierung von MALDI-TOF-MS-Methoden sollen die Ansätze der Leitlinie weiterhin in andere relevante Gremien eingebracht werden, um Normen in diesem Bereich aktiv mitzugestalten. Die Vorgehensweise der Validierung anhand einer umfangreichen, gut dokumentierten und durch mehrere Nutzer und Nutzerinnen gemeinschaftlich erarbeiteten Sammlung an Spektren lässt sich möglicherweise auch auf andere musterbasierte Identifizierungsmethoden übertragen.

Funding Open Access funding enabled and organized by Projekt DEAL.

\section{Declarations}

Conflict of interest Die Autoren erklären, dass sie keine Interessenskonflikte haben. The authors have no conflicts of interest to declare that are relevant to the content of this article.

Open Access This article is licensed under a Creative Commons Attribution 4.0 International License, which permits use, sharing, adaptation, distribution and reproduction in any medium or format, as long as you give appropriate credit to the original author(s) and the source, provide a link to the Creative Commons licence, and indicate if changes were made. The images or other third party material in this article are included in the article's Creative Commons licence, unless indicated otherwise in a credit line to the material. If material is not included in the article's Creative Commons licence and your intended use is not permitted by statutory regulation or exceeds the permitted use, you will need to obtain permission directly from the copyright holder. To view a copy of this licence, visit http://creativecommons.org/licenses/by/4.0/.

\section{Literatur}

BVL (2021) Leitlinien für die Validierung von Spezies-Identifizierungen mittels matrixunterstützter Laser-Desorptions-/IonisationsFlugzeit-Massenspektrometrie (MALDI-TOF-MS) im Einzellabor oder in Laborverbünden. https://www.bvl.bund.de/SharedDocs/ Downloads/07_Untersuchungen/Leitlinien-Validierung-von-Spezi es-Identifizierungen-MALDI-TOF-MS.pdf. Accessed 12 April 2021

DIN EN ISO 16140-1 bis -6 (2016) Normenreihe zur Mikrobiologie der Lebensmittelkette; Verfahrensvalidierung

Huber I, Pavlovic M, Maggipinto M, Konrad R, Busch U (2018) Interlaboratory proficiency test using MALDI-TOF MS for identification of food-associated bacteria. Food Anal Methods 11:10681075. https://doi.org/10.1007/s12161-017-1084-y

Malorny B, Scheel K, Rau J, Beyer W, Buschulte A, Nöckler K, Kreienbrock L (2020) Onlineumfrage zur Anwendung von molekularbiologischen Typisierungsverfahren und MALDI-TOF-MS in diagnostischen Laboren in Deutschland. J Consum Prot Food Saf 15:387-391. https://doi.org/10.1007/s00003-020-01297-8

Pavlovic M, Huber I, Konrad R, Busch U (2013) Application of MALDI-TOF MS for the identification of food borne bacteria. Open Microbiol J 7:135. https://doi.org/10.2174/1874285801 307010135

Pavlovic M, Huber I, Busch U (2020) MALDI-TOF MS profiling based identification of food components. Compr Foodomics. https://doi. org/10.1016/B978-0-08-100596-5.22767-4

Rau J, Eisenberg T, Männig A, Wind C, Lasch P, Sting R (2016) MALDI-UP - an internet platform for the exchange of MALDITOF mass spectra. Asp Food Control Anim Health 01:1-17

Rau J, Korte N, Dyk M, Wenninger O, Schreiter P, Hiller E (2020) Rapid animal species identification of feta and mozzarella cheese using MALDI-TOF mass-spectrometry. Food Control 117:107349. https://doi.org/10.1016/j.foodcont. 2020.107349

Rau J, Hiller E, Männig A, Dyk M, Wenninger O, Stoll P, Wibbelt G, Schreiter P (2021) Animal species identification of meat using MALDI-TOF MS. ChemRxiv Prepr. https://doi.org/10.26434/ chemrxiv.14229413.v1 
Rizzardi K, Wahab T, Jernberg C (2013) Rapid Subtyping of Yersinia enterocolitica by matrix-assisted laser desorption ionization-time of flight mass spectrometry (MALDI-TOF MS) for diagnostics and surveillance. J Clin Microbiol 51:4200-4203. https://doi.org/ 10.1128/JCM.01416-13

Stahl A, Schröder U (2017) Development of a MALDI-TOF MSbased protein fingerprint database of common food fish allowing fast and reliable identification of fraud and substitution. $\mathrm{J}$ Agric Food Chem 65:7519-7527. https://doi.org/10.1021/acs. jafc. $7 \mathrm{~b} 02826$

Szabo K, Grohmann L, Klemm C, Mierke-Klemeyer S, Reimann D, Franks K, Stoyke M (2017) § 64 LFGB Kick-off Meeting zu "Anwendungspotenzial moderner Analysetechniken im Bereich Lebensmittel- und Futtermittelsicherheit und deren Authentizität". J Verbrauch Lebensm 12:189-194. https://doi.org/10. 1007/s00003-017-1103-3

Trevethan R (2017) Sensitivity, specificity, and predictive values: foundations, pliabilities, and pitfalls in research and practice.
Front Public Health 5:307. https://doi.org/10.3389/fpubh.2017. 00307

Ulrich S, Gottschalk C, Dietrich R, Märtlbauer E, Gareis M (2019) Identification of cereulide producing Bacillus cereus by MALDITOF MS. Food Microbiol 82:75-81. https://doi.org/10.1016/j.fm. 2019.01.012

Verordnung (EU) 2017/625 (2017) des Europäischen Parlaments und des Rates vom 15. März 2017 über amtliche Kontrollen und andere amtliche Tätigkeiten zur Gewährleistung der Anwendung des Lebens- und Futtermittelrechts und der Vorschriften über Tiergesundheit und Tierschutz, Pflanzengesundheit und Pflanzenschutzmittel. Amtsbl Europ Union

Publisher's Note Springer Nature remains neutral with regard to jurisdictional claims in published maps and institutional affiliations. 